\title{
Pressure dependence of the Verwey transition in magnetite: an infrared spectroscopic point of view
}

\author{
J. Ebad-Allah, ${ }^{1}$ L. Baldassarre, ${ }^{1}$ M. Sing, ${ }^{2}$ R. Claessen, ${ }^{2}$ V. A. M. Brabers, ${ }^{2}$ and C. A. Kuntscher ${ }^{a)}$ \\ 1) Experimentalphysik 2, Universität Augsburg, D-86195 Augsburg, Germany \\ 2) Physikalisches Institut, Universität Würzburg, D-97074 Würzburg, Germany
}

(Dated: 30 October 2018)

We investigated the electronic and vibrational properties of magnetite at temperatures from $300 \mathrm{~K}$ down to $10 \mathrm{~K}$ and for pressures up to $10 \mathrm{GPa}$ by far-infrared reflectivity measurements. The Verwey transition is manifested by a drastic decrease of the overall reflectance and the splitting of the phonon modes as well as the activation of additional phonon modes. In the whole studied pressure range the down-shift of the overall reflectance spectrum saturates and the maximum number of phonon modes is reached at a critical temperature, which sets a lower bound for the Verwey transition temperature $\mathrm{T}_{\mathrm{v}}$. Based on these optical results a pressure-temperature phase diagram for magnetite is proposed.

PACS numbers: 78.30.-j,62.50.-p,71.30.+h

Keywords: magnetite, Verwey transition, high pressure, infrared spectroscopy

\section{INTRODUCTION}

Numerous experimental studies have been carried out on the natural mineral magnetite $\left(\mathrm{Fe}_{3} \mathrm{O}_{4}\right)$ to understand its puzzling electronic and magnetic properties. It is the oldest known magnetic material and the prototype material for the Verwey transition, which leads to charge ordering $\stackrel{1}{=} \mathrm{Fe}_{3} \mathrm{O}_{4}$ has an inverse cubic spinel structure at ambient conditions and is in a mixed-valence state described as $\left[\mathrm{Fe}^{3+}\right]_{\mathrm{A}}\left[\mathrm{Fe}^{2+}+\mathrm{Fe}^{3+}\right]_{\mathrm{B}} \mathrm{O}_{4}$, where $\mathrm{A}$ and $\mathrm{B}$ denote the tetrahedral and octahedral sites, respectively, in the spinel structure $\mathrm{AB}_{2} \mathrm{O}_{4}$, with space group $\mathrm{Fd} \overline{3} \mathrm{~m} .2$ At ambient conditions magnetite can be classified as a bad metal due to the presence of a small Drude contribution $\underline{3}^{\underline{3}} \underline{\underline{5}}$ With decreasing temperature magnetite undergoes a metal-to-insulator transition at the so-called Verwey transition temperature $\mathrm{T}_{\mathrm{v}} \approx 120 \mathrm{~K}$, concurrent with a lowering of the crystal structure symmetry from cubic to monoclinic. $\underline{\underline{6}-\underline{9}}$ Various experimental investigations led to contradictory scenarios for the rearrangement of charges on the Fe sites and the resulting charge ordering below $\mathrm{T}_{\mathrm{V}} \stackrel{10-15}{\underline{10}}$ In contrast, consistent results have been obtained by various groups ${ }^{3}-\underline{-5}$ regarding the changes in the optical properties when cooling below $\mathrm{T}_{\mathrm{v}}$ : According to infrared reflectivity measurements as a function of temperature a clear opening of a charge gap $(\approx 0.14 \mathrm{eV})$ and the appearance of numerous infrared modes due to the crystal symmetry lowering were observed.

Besides the charge ordering pattern below $T_{v}$, a matter of controversy is furthermore the behavior of $T_{v}$ as a function of pressure. Resistivity measurements 16,17 showed a sharp drop of $\mathrm{T}_{\mathrm{v}}$ at the critical pressure $P_{c}=7$ $8 \mathrm{GPa}$ to zero and a metallization above $P_{c}$. This find-

\footnotetext{
a) Electronic mail: christine.kuntscher@physik.uni-augsburg.de Department of Physics, Eindhoven University of Technology, 5600 MB Eindhoven, The Netherlands
}

ing was confirmed lateron by ac magnetic susceptibility together with resistivity measurements $\frac{18}{1}$ In Ref. 18 furthermore the existence of a quantum critical point in the pressure-temperature phase diagram of magnetite was claimed 18 In contradiction to these results, several groups found a linear decrease of $\mathrm{T}_{\mathrm{v}}$ with increasing pressure based on resistivity measurements, with a linear pressure coefficient of either $-2.8 \mathrm{~K} / \mathrm{GPa}^{19}-21$ or $-5 \mathrm{~K} / \mathrm{GPa}^{22.23}$. A recent Raman study confirmed the linear decrease of $\mathrm{T}_{\mathrm{v}}$ with $-5 \mathrm{~K} / \mathrm{GPa} .^{24}$ The absence or presence of a quantum critical point could be related to the level of hydrostaticity in the pressure cell, which may vary for the different types of experiments. Also, the measurement technique might play a role.

The above-mentioned controversy regarding $\mathrm{T}_{\mathrm{v}}(P)$ motivated us to study the Verwey transition in magnetite as a function of pressure by far-infrared reflectivity measurements, which comprise both electronic and vibrational properties. The goal of our investigation is not primarily the precise determination of the pressure dependence of the Verwey transition temperature like in other studies, but to characterize the phases of magnetite close to the Verwey transition from an infrared spectroscopic point of view: The overall level of the reflectance spectrum and the phonon mode activation and splitting are considered for various temperatures and pressures. Based on these two criteria we propose a pressure-temperature phase diagram. We also relate our results to those of earlier works.

\section{METHODS}

The single crystals of magnetite used in this work were grown from polycristalline $\mathrm{Fe}_{3} \mathrm{O}_{4}$ bars by using a floatingzone technique with radiation heating. ${ }^{25}$ The polycrystalline bars were prepared from $\alpha-\mathrm{Fe}_{2} \mathrm{O}_{3}$ by the usual ceramic procedures as pressing and sintering in an adapted oxygen atmosphere. The quality of the crystals was 


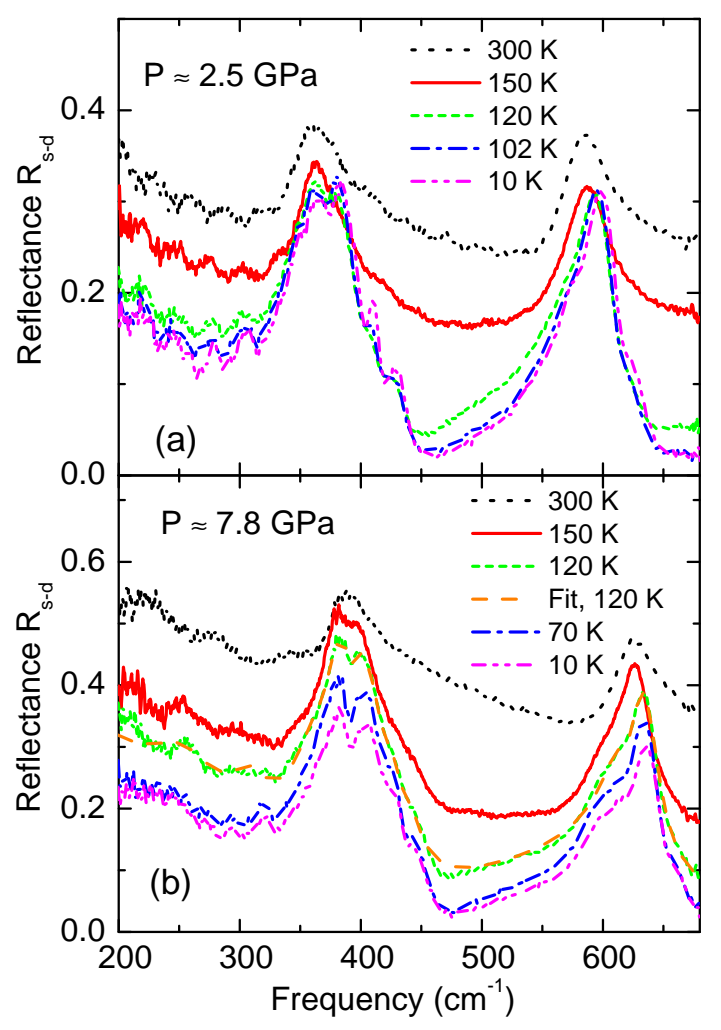

FIG. 1. Reflectance spectrum of magnetite for various temperatures at around (a) $2.5 \mathrm{GPa}$ and (b) $7.8 \mathrm{GPa}$. In (b) additionally the fitting curve based on the Drude-Lorentz model for the reflectance spectrum at $120 \mathrm{~K}$ is shown.

checked by electrical transport measurements, showing a sharp increase of the resistivity by a factor of about 100 at the critical temperature $\mathrm{T}_{\mathrm{v}} \approx 122 \mathrm{~K}$, which is characteristic for the Verwey transition. 26 The low-temperature reflectance measurements under pressure were conducted in the frequency range from 200 to $700 \mathrm{~cm}^{-1}$ with a frequency resolution $1 \mathrm{~cm}^{-1}$ partly at the infrared beamline of the synchrotron radiation source ANKA and partly in the lab at Augsburg university. A clamp diamond anvil cell (Diacell cryoDAC-Mega) equipped with type IIA diamonds, which are suitable for infrared measurements, was used for the generation of pressures up to $10 \mathrm{GPa}$. Finely ground CsI powder was used as quasi-hydrostatic pressure transmitting medium, in order to ensure a welldefined sample-diamond interface throughout the experiment. The pressure in the diamond anvil cell (DAC) was determined in situ in the cryostat by the ruby luminescence method ${ }^{27}$ The width of the phonon modes in the measured high-pressure reflectance spectra is comparable to that reported earlier on samples at ambient conditions,$\underline{\underline{3}}$ evidencing the quasi-hydrostatic conditions in the DAC.

The reflectance measurements at low temperature and high pressure were carried out using a home-built infrared microscope coupled to the FTIR spectrometer and main-

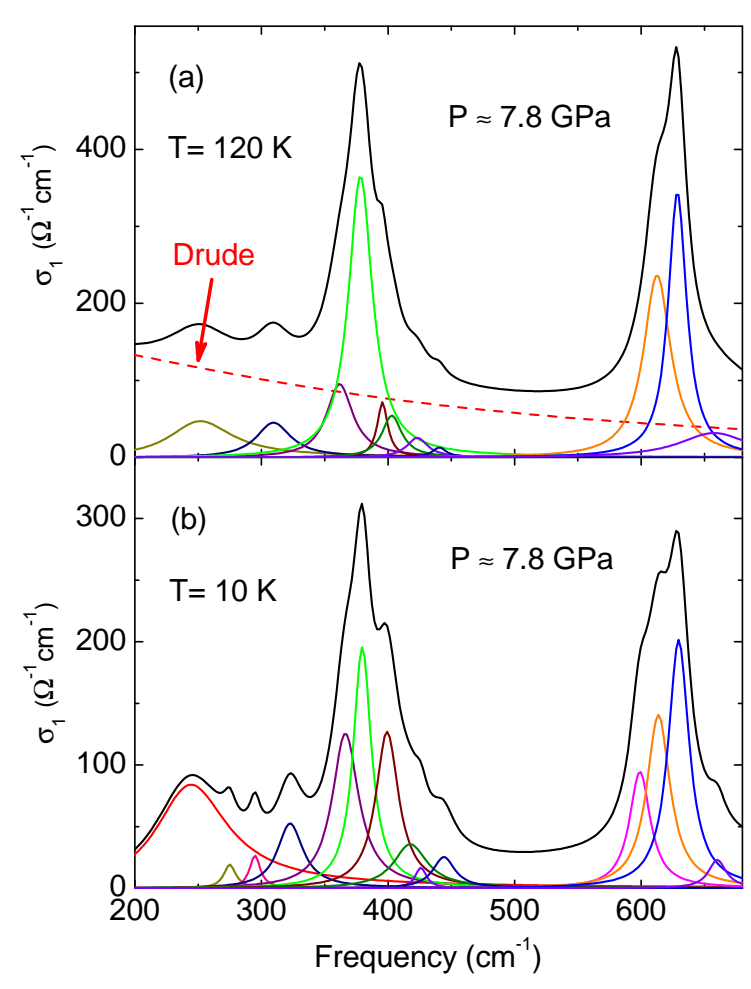

FIG. 2. Real part of the optical conductivity of magnetite at $7.8 \mathrm{GPa}$ and (a) $120 \mathrm{~K}$ and (b) $10 \mathrm{~K}$, obtained from the fitting of the reflectance spectra with the Drude-Lorentz model. The contributions (Lorentz oscillators; Drude term at $120 \mathrm{~K}$ ) based on the fitting are also shown.

tained at the same vacuum conditions, in order to avoid absorption lines of $\mathrm{H}_{2} \mathrm{O}$ and $\mathrm{CO}_{2}$ molecules. The infrared radiation was focused on the sample by all-reflecting Schwarzschild objectives with a large working distance of about $55 \mathrm{~mm}$ and $14 \mathrm{x}$ magnification. The DAC was mounted in a continuous-flow helium cryostat (Cryo Vac KONTI cryostat). More details about the geometry of the reflectivity measurements can be found in our earlier publications $\frac{28,29}{2}$ As reference, we used the intensity reflected from the $\mathrm{CuBe}$ gasket inside the DAC. All reflectance spectra shown in this paper refer to the absolute reflectance at the sample-diamond interface, denoted as $R_{\mathrm{s}-\mathrm{d}}$. Furthermore, corrections regarding the decaying intensity of the synchrotron radiation with time have been taken into account.

\section{RESULTS AND DISCUSSION}

The far-infrared reflectance spectrum of magnetite was measured for several pressures between 0 and $10 \mathrm{GPa}$ as a function of temperature. As an example, we show in Fig. 1 (a) the reflectance spectrum at $\approx 2.5 \mathrm{GPa}$ for various temperatures. At room temperature two oxygen phonon modes are observed with frequencies close to $355 \mathrm{~cm}^{-1}$ and $565 \mathrm{~cm}^{-1}$ at low pressure, consistent with 


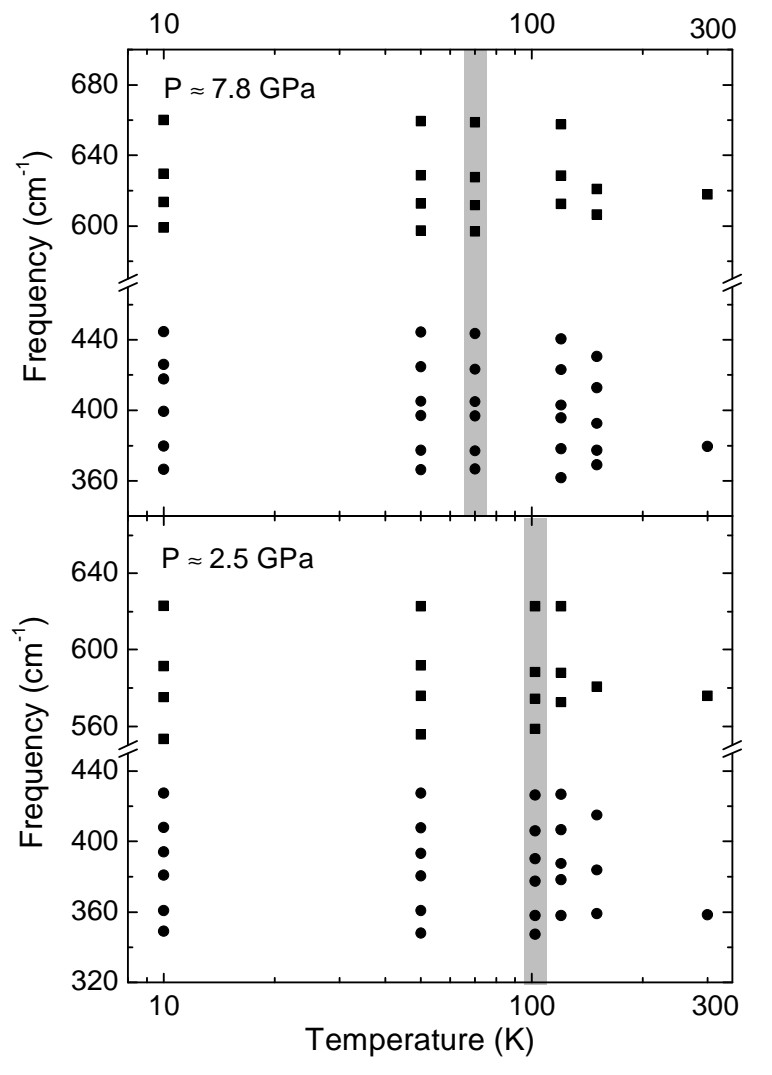

FIG. 3. Splitting of the two phonon modes and activation of phonon modes with decreasing temperature at 2.5 and 7.8 GPa. At a critical temperature (marked by a gray bar) the maximum number of phonon modes is reached.

earlier reports $\cdot \underline{3}-\underline{5}, 26,30$ While cooling down from room temperature to $150 \mathrm{~K}$, which is well above $\mathrm{T}_{\mathrm{v}}, \frac{17-24}{2}$ the overall reflectance spectrum gradually decreases [see Fig. 1(a)], consistent with a "bad metal" behavior also found in temperature-dependent dc resistivity data. ${ }^{26}$ Below $\approx 150 \mathrm{~K}$ the overall reflectance decreases drastically with a saturation below $\approx 102 \mathrm{~K}$. Furthermore, below $150 \mathrm{~K}$ the reflectance spectrum becomes more complex because of the phonon mode splitting and the activation of additional low-frequency phonon modes. By comparing the temperature dependence of the reflectance spectra for two pressures (2.5 and $7.8 \mathrm{GPa}$ ), as depicted in Figs. 1(a) and (b), it is obvious that this overall evolution of the reflectance spectrum with decreasing temperature is not strongly dependent on the applied pressure. Furthermore, at a fixed temperature the overall reflectance spectrum is enhanced with increasing pressure, which is consistent with the pressure-induced increase of the conductivity according to dc transport measurements $\underline{\underline{17}}$

For a quantitative characterization of the pressure- and temperature-induced changes in the optical response, the reflectance spectra were fitted according to the Fresnel equation for normal-incidence reflectivity taking into ac-

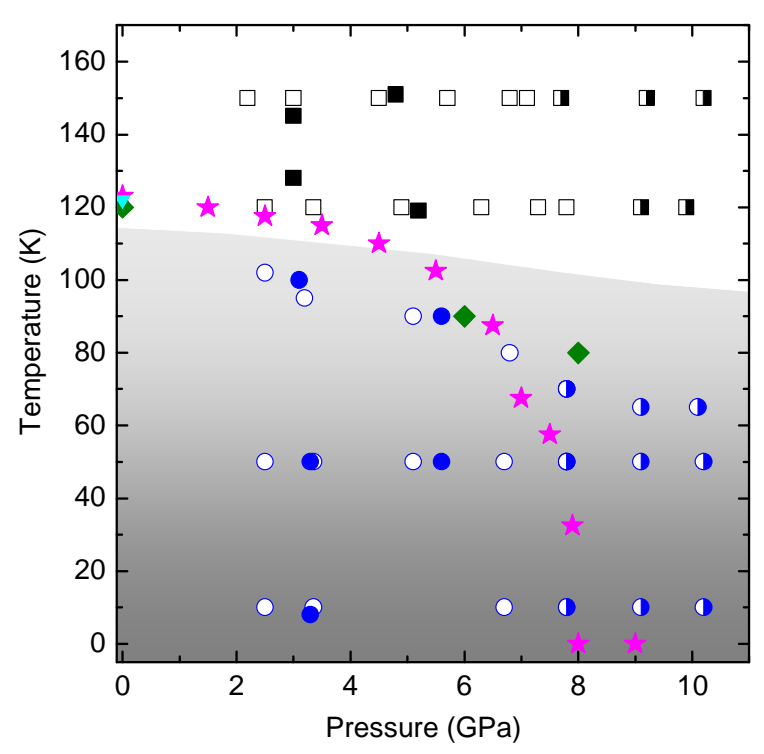

FIG. 4. Pressure-temperature phase diagram of magnetite for temperatures below $160 \mathrm{~K}$ and pressures up to $\approx 10 \mathrm{GPa}$. The squares correspond to the "bad metal" state and the circles to the insulating state. The results from three data sets are distinguished by empty, filled, and half-filled symbols. The gray region indicates the charge-ordered, insulating phase as observed by our optical data. The Verwey transition temperature based on earlier dc transport measurements at ambient pressure is marked by the filled down triangle. ${ }^{26}$ The results for the Verwey transition temperature $T_{\mathrm{v}}$ of Ref. 17 are marked by filled stars, and those of Ref. 24 by filled diamonds.

count the diamond-sample interface:

$$
R_{\mathrm{s}-\mathrm{d}}=\left|\frac{n_{\mathrm{dia}}-\sqrt{\epsilon_{\mathrm{s}}}}{n_{\mathrm{dia}}+\sqrt{\epsilon_{\mathrm{s}}}}\right|^{2} ; \quad \epsilon_{\mathrm{s}}=\epsilon_{\infty}+\frac{i \sigma}{\epsilon_{0} \omega}
$$

where $n_{d i a}$ is the refractive index of diamond and assumed to be independent of pressure and temperature, and $\epsilon_{\mathrm{s}}$ is the complex dielectric function of the sample. From the function $\epsilon_{\mathrm{s}}(\omega)$ the real part of the optical conductivity, $\sigma_{1}(\omega)$, can be calculated according to Eq. (11), where $\epsilon_{\infty}$ is the background dielectric constant (here $\left.\epsilon_{\infty} \approx 1\right) . \epsilon_{\mathrm{S}}(\omega)$ was assumed to follow the DrudeLorentz model .31 An example for the fitting is depicted in Fig. 1(a) for $\mathrm{T}=120 \mathrm{~K}$ at $P=7.8 \mathrm{GPa}$ : To obtain a good fit of the reflectance spectrum, we had to include a Drude term and several Lorentz oscillators describing the phonon modes, while the high-frequency extrapolation was modeled according to ambient-temperature data. ${ }^{26}$ It is important to note here that the extrapolations are not significant for our conclusions (see below) on the pressure-temperature phase diagram of magnetite. The various excitations (Drude term, phonon modes) at $\mathrm{T}=120 \mathrm{~K}$ and $7.8 \mathrm{GPa}$ are illustrated in Fig. 2 (a) together with the real part of the optical conductivity within the measured frequency range. At the lowest studied temperature $(10 \mathrm{~K})$ the optical conductivity can be 
described as a sum of Lorentz oscillators reflecting the rich phonon spectrum below $\mathrm{T}_{\mathrm{v}}$ [see Fig. 2 (b)].

As a criterium for entering the charge-ordered state one can take the saturation of the down-shift of the reflectance spectrum (see Fig. 1) and the absence of a Drude term. Concomitant with the overall lowering of the reflectance spectrum occurs the splitting of the phonon modes and the activation of additional modes. The mode splitting and activation are manifestations of symmetry lowering of the crystal structure (from cubic to monoclinic) at the Verwey transition $\stackrel{3}{-5}^{-5}$ Therefore, reaching the maximum number of phonon modes serves as an additional criterium for entering the charge-ordered phase. The temperature-dependent phonon spectrum was characterized based on the Drude-Lorentz fitting, and the extracted mode frequencies are shown in Fig. 3 at 2.5 and $7.8 \mathrm{GPa}$. Interestingly, the splitting and activation of phonon modes already start at around $150 \mathrm{~K}$, i.e., well above $\mathrm{T}_{\mathrm{v}}$, in agreement with earlier infrared studies. 5.32 The temperature at which a saturation regarding the down-shift of the reflectance spectrum and the number of phonon modes is reached sets a lower bound for $\mathrm{T}_{\mathrm{v}}$.

Based on two criteria - the down-shift of the reflectance spectrum and the phonon mode splitting/activation we can mark the "bad metal" and insulating phases in the pressure-temperature phase diagram of magnetite, as shown in Fig. 4. The most important result is that we do not observe a suppression of the insulating state even at the highest pressure applied (10 GPa), in agreement with some earlier studies. ${ }^{19-24}$ We do not observe the metallization of magnetite above $\approx 8 \mathrm{GPa}$ and the occurrence of a quantum critical point, as claimed in other works $16-18,33$ Whether or not this is due to the quasihydrostatic conditions in the DAC remains an open question, as no systematic study on the pressure dependence of the Verwey transition temperature in the same sample for various pressure transmitting media exists.

\section{CONCLUSION}

In conclusion, based on the temperature- and pressuredependent optical response of magnetite we find the typical signatures of the Verwey transition, namely (i) the overall lowering of the reflectance related to the entering of the charge-ordered state, and (ii) the splitting and activation of phonon modes due to the lowering of the crystal symmetry. The downshift of the reflectance spectrum and the splitting/activation of phonon modes are completed at a critical temperature, which sets a lower bound for $T_{\mathrm{v}}$. Based on these results, we propose a phase diagram for magnetite showing the "bad metal" and the insulating phases. The metallization of magnetite above $\approx 8 \mathrm{GPa}$ and the occurrence of a quantum critical point is not observed in our data.

\section{ACKNOWLEDGMENTS}

We acknowledge the ANKA Angströmquelle Karlsruhe for the provision of beamtime and thank B. Gasharova, Y.-L. Mathis, D. Moss, and M. Süpfle for assistance using the beamline ANKA-IR. We thank K. Syassen for providing valuable information about the construction of the home-made infrared microscope. This work was financially supported by the German Science Foundation (DFG) through SFB 484.

${ }^{1}$ E. J. W. Verwey, Nature (London) 144, 327 (1939).

${ }^{2}$ S. Sasaki, Acta Crystallogr. B 53, 762 (1997).

${ }^{3}$ L. Degiorgi, I. Blatter-Mörke, and P. Wachter, Phys. Rev. B 35, $5421(1987)$.

${ }^{4}$ S. K. Park, T. Ishikawa, and Y. Tokura, Phys. Rev. B 58, 3717 (1998).

${ }^{5}$ L.V. Gasparov, D. B. Tanner, D. B. Romero, H. Berger, G. Margaritondo, and L. Forró, Phys. Rev. B 62, 7939(2000).

${ }^{6}$ M. Iizumi, Acta Crystallogr. Sect. B 38, 2121 (1982).

${ }^{7}$ J. P. Wright, J. P. Attfield, and P. G. Radaelli, Phys. Rev. Lett. $\mathbf{8 7}, 266401$ (2001);

${ }^{8}$ J. P. Wright, J. P. Attfield, and P. G. Radaelli, Phys. Rev. B 66, 214422 (2002).

${ }^{9}$ M. S. Senn, J. P. Wright, and J. P. Attfield, Nature 481, 173 (2012).

${ }^{10}$ E. Nazarenko, J. E. Lorenze, Y. L. Hodeau, D. Mannix, and C. Marinl, Phys. Rev. Lett. 97, 056403 (2006).

${ }^{11}$ H. Kobayashi, Phys. Rev. B 73, 104110 (2006).

${ }^{12}$ M. P. Pasternak, W. M. Xu, G. Kh. Rozenberg, R. D. Taylor, and R. Jeanloz, Magn. Magn. Mater. 265, L107 (2003).

${ }^{13}$ G. Kh. Rozenberg, Y. Amiel, W. M. Xu, M. P. Pasternak,R. Jeanloz, M. Hanfland, and R. D. Taylor, Phys. Rev. B 75, 020102 (2007).

${ }^{14}$ S. V. Ovsyannikov, V. V. Shchennikov, S. Todo and Y. Uwatoko, J. Phys.: Condens. Matter 20, 172201 (2008).

${ }^{15}$ F. Baudelet, S. Pascarelli, O. Mathon, J.-P. Itie, A. Polian, and J.-C. Chervin, Phys. Rev. B 82, 140412(R) (2010).

${ }^{16} \mathrm{~S}$. Todo, N. Takeshita, T. Kanehara, T. Mori, and N. Môri, J. Appl. Phys. 89, 7347 (2001).

${ }^{17}$ N. Môri, S. Todo, N. Takeshita, T. Mori, and Y. Akishige, Physica B 312-313, 686 (2002).

${ }^{18}$ J. Spalek, A. Kozlowski, Z. Tarnawski, Z. Kakol, Y. Fukami, F. Ono, R. Zach, L. J. Spalek, and J. M. Honig, Phys. Rev. B 78, 100401(R) (2008).

${ }^{19}$ Y. Kakudate, N. Môri, and Y. Kino, J. Magn. Magn. Mater. 12, $22(1979)$.

${ }^{20}$ S. Tamura, J. Phys. Soc. Jpn. 59, 4462 (1990).

${ }^{21}$ G. K. Rozenberg, G. R. Hearne, M. P. Pasternak, P. A. Metcalf, and J. M. Honig, Phys. Rev. B 53, 6482 (1996).

${ }^{22}$ G. A. Samara, Phys. Rev. Lett. 21, 795 (1968).

${ }^{23}$ S. K. Ramasesha, M. Mohan, A. K. Singh, J. M. Honig, and C. N. R. Rao, Phys. Rev. B 50, 13789 (1994).

${ }^{24}$ L. V. Gasparov, D. Arenas, K.-Y. Choi, G. Günteroth, H. Berger, L. Forro, G. Margaritondo, V. V. Struzhkin, and R. Hemley, J. Appl. Phys. 97, 10A922 (2005).

${ }^{25}$ V. A. M. Brabers, J. Cryst. Growth 8, 26 (1971).

${ }^{26}$ J. Ebad-Allah, L. Baldassarre, M. Sing, R. Claessen, V. A. M. Brabers, and C. A. Kuntscher, arXiv:1202.4431 (unpublished).

${ }^{27}$ H. K Mao, J. Xu, and P.M. Bell, J. Geophys. Res. 91, 4673 (1986).

${ }^{28}$ A. Pashkin, M. Dressel, and C. A. Kuntscher, Phys. Rev. B 74, 165118 (2006).

${ }^{29}$ C. A. Kuntscher, S. Frank, A. Pashkin, M. Hoinkis, M. Klemm, M. Sing, S. Horn, and R. Claessen, Phys. Rev. B 74, 184402 (2006). 
${ }^{30}$ J. Ebad-Allah, L. Baldassarre, M. Sing, R. Claessen, V. A. M. Brabers, and C. A. Kuntscher, High Pressure Research 29, 500 (2009).

${ }^{31} \mathrm{~F}$. Wooten, Optical properties of solids (Academic Press, New York, 1972)

${ }^{32}$ A. Pimenov, S. Tachos, T. Rudolf, A. Loidl, D. Schrupp, M. Sing, R. Claessen, and V. A. M. Brabers, Phys. Rev. B 72, 035131
(2005).

${ }^{33}$ G. Kh. Rozenberg, M. P. Pasternak, W. M. Xu, Y. Amiel, M. Hanfland, R. D. Taylor, and R. Jeanloz, Phys. Rev. Lett. 96, 045705 (2006). 